\title{
Damage Localization of an Offshore Platform considering Temperature Variations
}

\author{
Shuqing Wang, Min Zhang, and Huajun Li \\ College of Engineering, Ocean University of China, Qingdao 266100, China \\ Correspondence should be addressed to Shuqing Wang; shuqing@ouc.edu.cn \\ Received 14 June 2015; Revised 4 September 2015; Accepted 6 September 2015 \\ Academic Editor: Yuri Vladimirovich Mikhlin
}

Copyright (c) 2015 Shuqing Wang et al. This is an open access article distributed under the Creative Commons Attribution License, which permits unrestricted use, distribution, and reproduction in any medium, provided the original work is properly cited.

\begin{abstract}
Modal parameters are sensitive indicators of structural damages. However, these modal parameters are sensitive not only to damage, but also to the environmental variations. Development of vibration based damage detection methodology which is robust to environmental variation is essentially important for the structural safety. The present paper utilizes a recently developed modal strain energy decomposition (MSED) method to localize the damage of an offshore structure. A progress of the present paper is to take the temperature variation into consideration and Monte Carlo simulation is introduced to investigate the effect of temperature variation on the robustness of damage localization. Numerical study is conducted on an offshore platform structure considering the temperature variation. Several damage cases, including single and double damage scenarios, are included to investigate the damage localization algorithm. Results indicate that the MSED algorithm is able to detect the damage despite the temperature variations.
\end{abstract}

\section{Introduction}

Vibration-based damage assessment and structural health monitoring (SHM) have been widely investigated during the past decades $[1,2]$. The essential feature of vibrationbased damage detection is that the dynamic characteristics of the structure would change due to the physical properties alteration of the structures. Such variations can be detected by utilizing modal identification methods and by monitoring the changes in the structure on a global basis.

Among the vibration based damage assessment methods, one type of damage identification methods only uses modal frequency information for damage assessment. Cawley and Adams [3] were among the first to use an incomplete set of natural frequencies to identify the location of damage. A detailed discussion on the use of natural frequency as a diagnostic parameter in structural assessment procedure can be found in the review paper by Salawu [4]. More recently, Wang and Li [5] and Wang [6] proposed an iterative modal strain energy method for damage localization and severity estimation, only requiring the changes of a few lower natural frequencies. Gillich and Praisach [7] presented a new method, based on natural frequency changes, able to detect damages in beam-like structures and to assess their location and severity, by considering the particular manner in which the natural frequencies of the weak-axis bending vibration modes change due to the occurrence of discontinuities. The advantages of frequency based methods include availability of modal frequencies from only one sensor record, and the identified frequencies have higher accuracy and robustness than those of mode shapes. However, information from only structural frequency is not sufficient, because modal frequency is a global property of structure and changes of frequency due to local damages may not be reflected, especially for large-scale structures such as offshore platforms.

Another class of damage diagnosis methods uses mode shapes. Early studies focus primarily on modal assurance criteria (MAC) and coordinate MAC (COMAC) values to identify damage by determining the level of correlation between modes from the test of undamaged and damaged structures [8-10]. Later, mode shape derivatives, such as mode shape curvature and strain-based mode shapes, were used as an alternative to obtain spatial information about vibration changes [11, 12]. After that, many methods based on dynamically measured flexibility have been proposed for damage detection. Because of the inverse relationship to the square of the modal frequencies, the measured flexibility matrix is most sensitive to changes in the lower-frequency modes of 
the structure [13-15]. However, mass-normalized mode shapes are required when formulating the flexibility matrix. Among the damage identification methods using mode shapes, the modal strain energy based methods seem to be promising for damage evaluation.

The Stubbs damage index algorithm, based on the decrease in modal strain energy, is defined by the curvature of the measured mode shapes $[12,16]$. This method requires that the mode shapes before and after damage be known, but the modes do not need to be mass normalized and only a few modes are required. More recently, modal strain energy decomposition (MSED) method has been developed for damage localization [17-19]. The MSED method defines two damage indicators, axial damage indicator and transverse damage indicator, for each member. Analyzing the joint information of the two damage indicators can greatly improve the accuracy of damage localization.

In vibration-based damage identification and SHM, dynamic modal parameters, such as natural frequencies and mode shapes, of a structure are usually used as damage-sensitive features. However, these modal parameters are not only sensitive to structural damage, but also to the environmental factors and operational conditions such as temperature, humidity, and traffic conditions [20-22]. The structural modal parameters may change along with the environmental factors and operational conditions, which could possibly mask the subtle structural changes caused by damage. Therefore, the influence of environmental effects should be recognized and eliminated before applying damage identification methods $[23,24]$. Several researchers have emphasized the importance of removing environmental or/and operational variations and have proposed some data cleansing procedures for SHM [20, 25-30]. Generally, three classes of methodologies, theoretical derivation methods, trend analysis methods, and methods of quantitative models, have been developed to correlate the temperatures and vibration properties. The interested reader can find more information in the two recently published review articles [21, 22].

From the literature review, one can see that a lot of papers concerning variations in vibration properties of civil structures under changing temperature conditions have been published. However, most of these studies focus on variations in frequencies of bridge structures, with some studies on variations in mode shapes and damping of bridge structures. Offshore platforms, operated in harsh environment and under various external forces, constantly accumulate damage during their service life. Clearly the development of robust techniques for early damage identification is crucial to avoid the possible occurrence of a catastrophic structural failure. For offshore structures, factors that influence the modal parameters cover variations of temperature, tidal level, foundation scouring, marine growth, and deck mass, and so forth. Modal changes produced by environmental conditions can be equivalent or greater than the ones produced by damage [31]. Therefore, development of robust damage assessment methodology, where the damage indicator is insensitive to environmental variations, has become to be a key problem in the damage detection and structural health monitoring. Generally there are two approaches which are able to handle such cases. One way is to firstly correlate the environmental variation and the modal parameters and conduct the damage assessment after the effect of environmental variation has been removed. Another more promising way is to develop a robust damage indicator, which is sensitive to structural damage but insensitive to environmental variations.

The objective of the present paper is to investigate the robustness of environmental variation on damage localization of offshore platform structures. A recently developed modal strain energy decomposition method is used for damage localization of an offshore platform structure. A progress of this paper is to take the temperature variation into consideration and Monte-Carlo simulation is introduced to investigate the effect of temperature variation on the damage localization. Different temperature variations for structural members which are located in air and beneath water are assumed. Both single damage and double damage scenarios are considered. To this end, the paper is structured as follows. In Section 2, the modal strain energy decomposition method for damage localization is briefly described. The model of a platform structure and simulations of environmental variation are introduced in details in Section 3. Section 4 reports and discusses the results of environmental variation on the damage localization. Finally, the paper is concluded with some final remarks in Section 5.

\section{Modal Stain Energy Decomposition Method for Damage Localization}

In this section, the modal strain energy decomposition method is briefly reviewed and will be used for damage localization [17-19].

Stubbs et al. [12] proposed a damage index for damage localization. The damage indicator, named Stubbs' damage index, is defined as

$$
\beta_{j}=\frac{E_{j}}{E_{j}^{*}}=\frac{\sum_{i=1}^{m}\left(\gamma_{i j}^{*}+\gamma_{i}^{*}\right) \gamma_{i}}{\sum_{i=1}^{m}\left(\gamma_{i j}+\gamma_{i}\right) \gamma_{i}^{*}},
$$

where $E_{j}$ and $E_{j}^{*}$ are Young's modulus for the $j$ th element before and after damage, respectively; $m$ is the number of modes being used; the elemental and global modal strain energy for the $i$ th mode shape is, respectively, defined as

$$
\begin{aligned}
\gamma_{i j} & =\Phi_{i}^{T} \mathbf{K}_{j 0} \Phi_{i}, \\
\gamma_{i} & =\Phi_{i}^{T} \mathbf{K}_{0} \Phi_{i}, \\
\gamma_{i j}^{*} & =\Phi_{i}^{* T} \mathbf{K}_{j 0} \Phi_{i}^{*}, \\
\gamma_{i}^{*} & =\Phi_{i}^{* T} \mathbf{K}_{0} \Phi_{i}^{*},
\end{aligned}
$$

in which $\Phi_{i}$ and $\Phi_{i}^{*}$ are the $i$ th mode shape of the undamaged and damaged structure, respectively; the superscript " $T$ " is the transpose operator and superscript "** is used to indicate a damage version. $\mathbf{K}_{j 0}=\mathbf{K}_{j} / E_{j}$, where $\mathbf{K}_{j}$ is the stiffness matrix of the $j$ th element defined in global coordinates for the undamaged system. $\mathbf{K}_{0}$ is the global stiffness matrix, which is constituted of $\mathbf{K}_{j 0}$ for all the finite elements. 
For a more robust classification criterion of damage location, a normalized damage indicator is defined as

$$
Z_{j}=\frac{\beta_{j}-\bar{\beta}}{\sigma_{\beta}}
$$

where $\bar{\beta}$ and $\sigma_{\beta}$ represent the mean and the standard deviation of $\beta_{j}$, respectively.

The major concept of the modal strain energy decomposition method is to separate the structural modal strain energy into two groups according to local element coordinates. One is axial modal strain energy and the other is transverse modal strain energy. Then two damage indicators can be defined [18]. For an elemental stiffness matrix $k_{j}$, it can be decomposed into

$$
k_{j}=k_{j}^{a}+k_{j}^{t}+k_{j}^{r}+k_{j}^{t r}
$$

where superscripts $a, t, r$, and $t r$ stand for axial, transverse, rotational, and transverse-rotational terms, respectively. That is, $k_{j}^{a}$ is the matrix containing axial stiffness terms only, $k_{j}^{t}$ is associated with the transverse stiffness terms only. It is recognized that the measurements associated with rotational coordinates are difficult to obtain practically, so most damage detection methods use mode shapes that include only translational coordinates.

Following the formulation of Stubbs index, the axial damage index can be obtained as follows:

$$
\beta_{j}^{a}=\frac{\sum_{i=1}^{m}\left(\Phi_{i}^{* T} \mathbf{K}_{j 0}^{a} \Phi_{i}^{*}+\Phi_{i}^{* T} \mathbf{K}_{0}^{a} \Phi_{i}^{*}\right) \Phi_{i}^{T} \mathbf{K}_{0}^{a} \Phi_{i}}{\sum_{i=1}^{m}\left(\Phi_{i}{ }^{T} \mathbf{K}_{j 0}^{a} \Phi_{i}+\Phi_{i}^{T} \mathbf{K}_{0}^{a} \Phi_{i}\right) \Phi_{i}^{* T} \mathbf{K}_{0}^{a} \Phi_{i}^{*}}
$$

In (8), $\mathbf{K}_{j}^{a}=E_{j} \mathbf{K}_{j 0}^{a}$ where $\mathbf{K}_{j}^{a}$ is defined as matrix $k_{j}^{a}$ transformed from local to global coordinates. And the matrix $\mathbf{K}_{j 0}^{a}$ involves only geometric quantities. Equation (8) can be viewed as the counterpart of (1) while only the axial modal strain energy is under consideration.

By the same token, if only the transverse modal strain energy is considered, one should obtain the corresponding index as

$$
\beta_{j}^{t}=\frac{\sum_{i=1}^{m}\left(\Phi_{i}^{* T} \mathbf{K}_{j 0}^{t} \Phi_{i}^{*}+\Phi_{i}^{* T} \mathbf{K}_{0}^{t} \Phi_{i}^{*}\right) \Phi_{i}{ }^{T} \mathbf{K}_{0}^{t} \Phi_{i}}{\sum_{i=1}^{m}\left(\Phi_{i}{ }^{T} \mathbf{K}_{j 0}^{t} \Phi_{i}+\Phi_{i}{ }^{T} \mathbf{K}_{0}^{t} \Phi_{i}\right) \Phi_{i}^{* T} \mathbf{K}_{0}^{t} \Phi_{i}^{*}}
$$

In (9), $\mathbf{K}_{j 0}^{t}$ is the geometric part of stiffness matrix corresponding to transverse parts in global coordinates.

Following the same normalization procedure as in (6), one can define the axial damage indicator as

$$
Z_{j}^{a}=\frac{\beta_{j}^{a}-\overline{\beta^{a}}}{\sigma_{\beta^{a}}}
$$

and the transverse damage indicator as

$$
Z_{j}^{t}=\frac{\beta_{j}^{t}-\overline{\beta^{t}}}{\sigma_{\beta^{t}}}
$$

where the overline represents the mean value and $\sigma$ represents the standard deviation of the corresponding variable.

The damage pattern is classified via a statistical-patternrecognition technique using hypothesis testing. Then the decision rule for damage location is selected as follows [32]. The structure is not damaged at the $j$ th location when $Z_{j}<$ $Z_{c}$ and is damaged when $Z_{j} \geq Z_{c}$ in which $Z_{c}$ is a number that reflects the level of significance of the test (e.g., if $Z_{c}=2$, then the confidence level is $97.7 \%$ ). It should be mentioned that $Z_{c}=2$ is used in the following numerical study for damage location.

A typical 3D frame structure generally consists of vertical columns, horizontal beams, and diagonal braces. If the vibration modes used in damage detection are mainly lateral (horizontal) motion, the modal strain energy of vertical members would be dominated by the transverse modal strain energy. On the other hand, the axial modal strain energy would contribute significantly to the modal strain energy of horizontal members and diagonal braces. Therefore, analyzing the joint information of the two damage indicators can greatly improve the accuracy of damage localization. For detailed explanation and comparison, the reader may refer to two published articles for more information $[18,19]$.

\section{Numerical Example}

3.1. Description of the Structure. An offshore platform structure described in [33] is used here for numerical study. The structure consists of 36 steel tubular members that comprise 12 leg members, 12 horizontal brace members, and 12 diagonal brace members in vertical planes. All members have uniform outer diameter $17.8 \mathrm{~cm}$ and wall thickness $0.89 \mathrm{~cm}$. The heights of the three stories are all $9.14 \mathrm{~m}$, and the side lengths of the bottom and top floors are $12.19 \mathrm{~m} \times$ $10.97 \mathrm{~m}$ and $4.88 \mathrm{~m} \times 3.66 \mathrm{~m}$, respectively. The essential material properties of the steel tubular members are elastic modulus $E=2.06 \times 10^{11} \mathrm{~N} / \mathrm{m}^{2}$, volumetric mass density $\rho=7850 \mathrm{~kg} / \mathrm{m}^{3}$, and Poisson's ratio $v=0.3$. The finite element model of the platform structure is synthesized, where each structural member is modeled as a three-dimensional uniform beam element, and is distinguished by assigning an element number. Also shown in Figure 1 are the node and element numbering, respectively. Modal analysis is carried out by developing a program in MATLAB environment to get the modal frequencies and mode shapes. The first three modal frequencies are $7.72,8.31$, and $8.87 \mathrm{~Hz}$, respectively. Also the first two mode shapes are exhibited in Figure 2, where the first mode is vibrated dominantly in the $y$-(short-span) direction and the second mode in the $x$-(long-span) direction. And the third mode is a torsion mode around $z$-axis. For simplicity, the still water level (SWL) is assumed to be exactly located on the second floor from the top, as shown in Figure 1.

3.2. Simulation of the Environmental Temperature. As described in Section 1, the variation of the environmental temperature will influence the physical properties of the structure. To consider the effect of temperature variation on the modal parameters, it is assumed that Young's modulus is dependent on the temperature. Woon and Mitchell [34] 


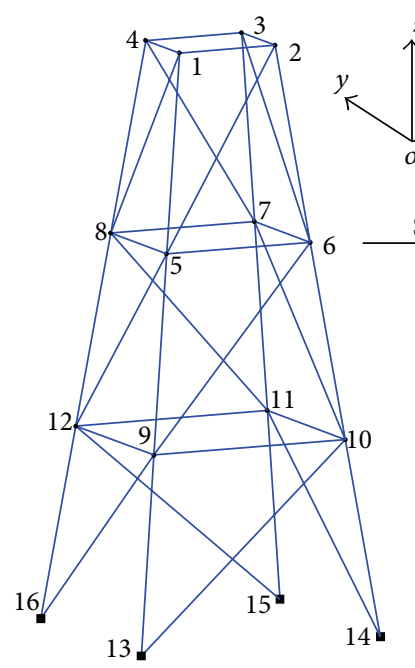

(a) Node numbering

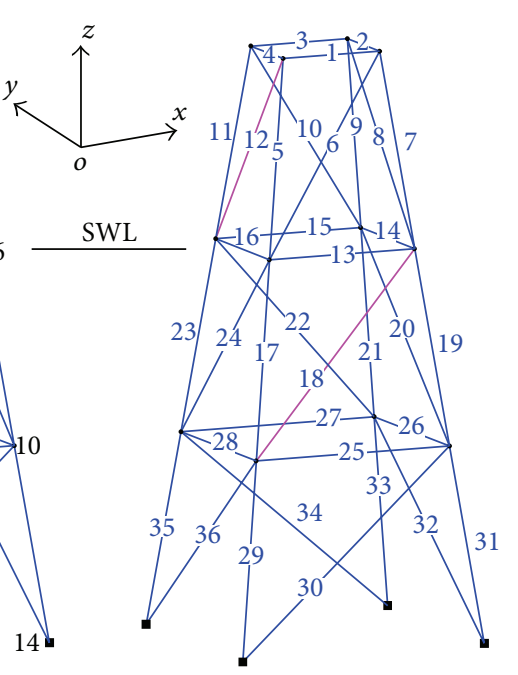

(b) Element numbering
FIGURE 1: Sketch of the offshore platform structure: (a) node numbering and (b) element numbering.
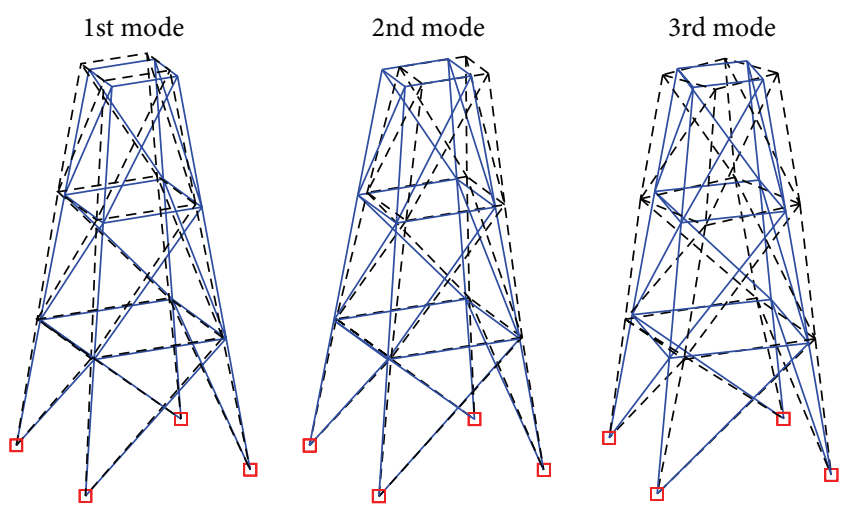

FIgURE 2: The first three mode shapes of the undamaged structure.

showed that $E(T(t))$ depends linearly on the temperature through experimental investigation; that is,

$$
E(T(t))=E\left(T^{0}\right)+\beta\left(T(t)-T^{0}\right),
$$

where $\beta$ is a temperature gradient of $E(T(t))$ that describes the linear change due to the temperature difference and $E\left(T^{0}\right)$ is Young's modulus at the reference ambient temperature. In this paper, $T^{0}$ is selected as $10^{\circ} \mathrm{C}$ and $E\left(T^{0}\right)$ is equal to $2.06 \times$ $10^{11} \mathrm{~N} / \mathrm{m}^{2}$. And the temperature gradient $\beta$ equals $-1.0 \times 10^{8}$, which means a linear decrease with the temperature increase.

To investigate the effect of temperature variations on damage localization, the air temperatures is considered a Gaussian process with mean $10^{\circ} \mathrm{C}$ and standard deviation $8^{\circ} \mathrm{C}$, and the water temperature is considered a Gaussian process with mean $15^{\circ} \mathrm{C}$ and standard deviation $4^{\circ} \mathrm{C}$. With this assumption, it indicates that the air temperature has a broader change range than that of the water temperature. At the same time, the average water temperature is higher than that of air temperature. In the following investigation, a 1000 Monte Carlo simulation was used to investigate
TABLE 1: Damage scenarios in the numerical study.

\begin{tabular}{lcccc}
\hline Damage case & $\begin{array}{c}\text { Damaged } \\
\text { member }\end{array}$ & $\begin{array}{c}\text { Damage } \\
\text { severity }\end{array}$ & $\begin{array}{c}1 \text { st } \\
\text { frequency } \\
(\mathrm{Hz})\end{array}$ & $\begin{array}{c}\text { 2nd } \\
\text { frequency } \\
(\mathrm{Hz})\end{array}$ \\
\hline Baseline & None & 0 & 7.7243 & 8.3101 \\
A & $y$-brace 12 & $30 \%$ & 7.6889 & 8.3100 \\
B & $x$-brace 18 & $20 \%$ & 7.7235 & 8.2547 \\
C & $y$-brace 12 & $30 \%$ & 7.6881 & 8.2547 \\
\hline
\end{tabular}

the effect of temperature variation on the robustness of damage localization. The histogram of the simulated temperature and its theoretical probability density function (PDF) are illustrated in Figure 3.

As described in Section 3.1, it is assumed that the still water level (SWL) is located on the second floor from the top, as shown in Figure 1. And it is assumed that this water level is not changed during the simulation. Under such circumstances, structural members 1-12 are assumed to be located in air and the others are beneath the water surface.

3.3. Damage Scenarios. In this paper, Young's modulus of the damaged members is reduced in order to obtain the modal parameters of the damaged structure by repeating the modal analysis. Three damage cases are synthesized for the numerical studies. The damaged members are illustrated in Figure 1. In damage case A, structural member 12 (for simplicity, named $y$-brace), which is orientated diagonally in yoz plane above SWL, is assumed to be damaged with a $30 \%$ stiffness loss. In damage case B, structural member 18 (named $x$-brace), a slanted diagonal member located beneath water and orientated in $x o z$ plane, is assumed to be damaged with a $20 \%$ stiffness reduction. Damage case $\mathrm{C}$ is a double damage scenario, where both of the structural members inflicted in damage cases $\mathrm{A}$ and $\mathrm{B}$ are included.

Table 1 summarizes the three damage scenarios. Since only the first two modes are utilized for damage localization of offshore platforms in Section 4, therefore, the first two modal frequencies of the baseline and the damaged structures are also listed in Table 1. It should be noted that Young's modulus of the baseline structure is set to be $2.06 \times 10^{11} \mathrm{~N} / \mathrm{m}^{2}$, which corresponds to a $10^{\circ} \mathrm{C}$ constant temperature. From Table 1 , it is noticed that the first frequency of the structure $B$ with a damaged $x$-brace at 18 and the second frequency of the structure A with a damaged $y$-brace at 12 do not change noticeably from their counterparts of the undamaged structure. This also suggests that a damaged $x$-brace does not have discernible effect on the first mode shape, which predominantly vibrates in the short-span $y$ direction. Likewise, a damaged short-span $y$-brace would have negligible effect on the second mode shape that vibrates in the $x$ direction.

\section{Results and Analysis}

4.1. Damage Localization of the Structure under No Temperature Variation. First, the damage localization without considering the temperature variations will be investigated. 


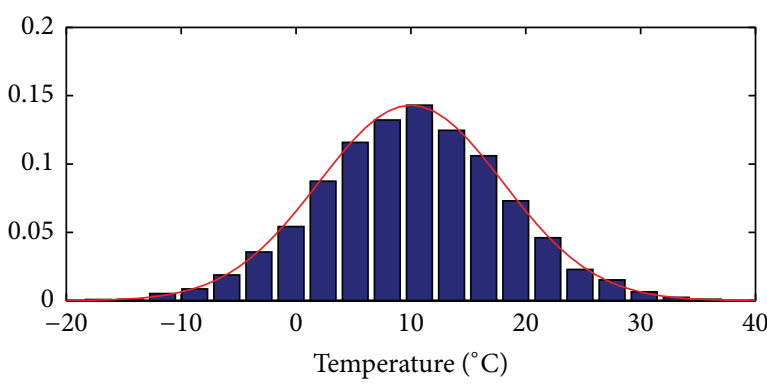

(a) Temp. PDF in air

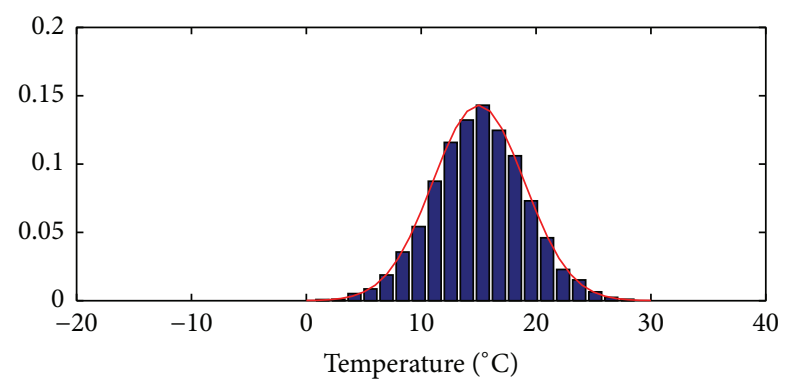

(b) Temp. PDF in water

FIGURE 3: Temperature variations used in the present paper.

The relative change ratios of the first modal frequency in damage case $\mathrm{A}$ and the second modal frequency in damage case $\mathrm{B}$ are $-0.46 \%$ and $-0.67 \%$, respectively. For the comparison of the mode shapes, the function of modal assurance criterion (MAC) can be used as a measure of degree of consistency (linearity) between one modal and another reference modal vector as follows:

$$
\operatorname{MAC}\left\{\Phi_{i}^{*}, \Phi_{i}\right\}=\frac{\left|\Phi_{i}^{* T} \Phi_{i}\right|^{2}}{\left(\Phi_{i}^{* T} \Phi_{i}^{*}\right)\left(\Phi_{i}^{T} \Phi_{i}\right)} .
$$

If the MAC has a unity value, this is an indication that the modal vectors are fully consistent. In the present study, the MACs for the first three modes are $0.9998,1.000$, and 0.9999 for damage case A and 0.9999, 0.9992, and 0.9994 for damage case $\mathrm{B}$, respectively. It is noticed that a damaged $y$-brace 12 in damage case A will only produce effect on the first mode shape, and a damaged $x$-brace 18 in damage case B will only produce effect on the second mode shape. At the same time, both damaged $y$-brace and $x$-brace have effect on the third mode which is a torsion mode shape. It should also be mentioned that a damaged structural member located in lower level (e.g., $x$-brace 18 in this study) have larger effect on the vibration properties than that produced by a damaged structural member that is located in upper level (e.g., $x$-brace 12).

As (8) and (9) show, the damage indicators do not utilize modal frequencies but mode shapes for damage localization. Throughout this numerical example, the first two mode shapes are utilized in the damage assessment. It has been verified that the axial indicator is better for localizing damaged brace member in both single and multiple damage cases [19]. Therefore, the axial damage indicator is used for damage localization in this paper. Shown in Figures 4 and 5 are the axial damage indicators for damage cases $\mathrm{A}$ and $\mathrm{C}$, respectively, when the first two mode shapes are utilized in the damage assessment. As illustrated in Figure 4, the damage indicator corresponding to element location 12 possesses the largest value (5.2230), which is much greater than the threshold $\left(Z_{c}=2\right.$ in this paper, as described in Section 2). And damage indicators associated with other elements are all less than 2 . Therefore, it can be concluded that element number 12 is the true damaged location. Similar results can be obtained for damage case B and the results are not shown here for space limitation. For the double damage scenario, the damage

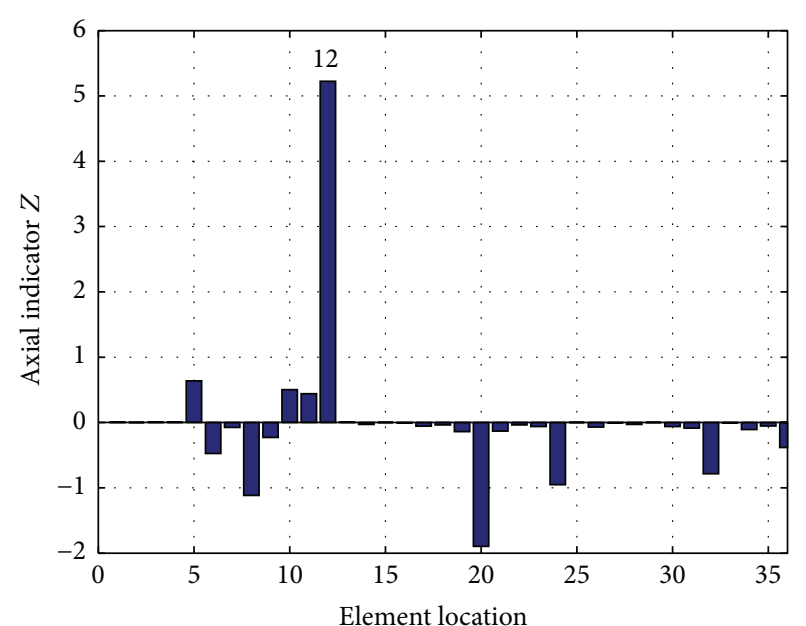

FIgURE 4: The damage indicator for damage case A.

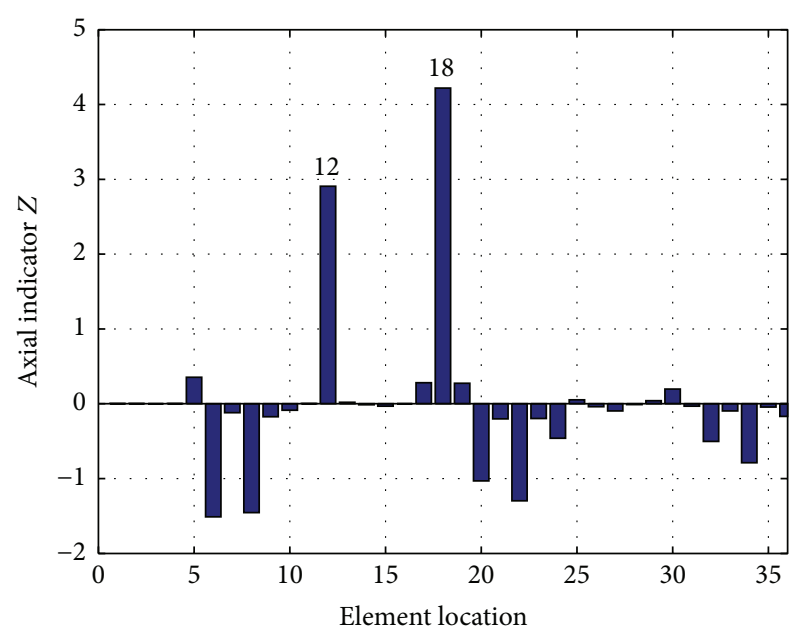

FIgURE 5: The damage indicator for case C.

localization is shown in Figure 5. It is obvious that the damage indicators associated with element numbers 12 and 18 are greater than 2, indicating that elements numbers 12 and 18 are damaged. From Figures 4 and 5, one can see that the present method is able to determine the damage locations correctly. 


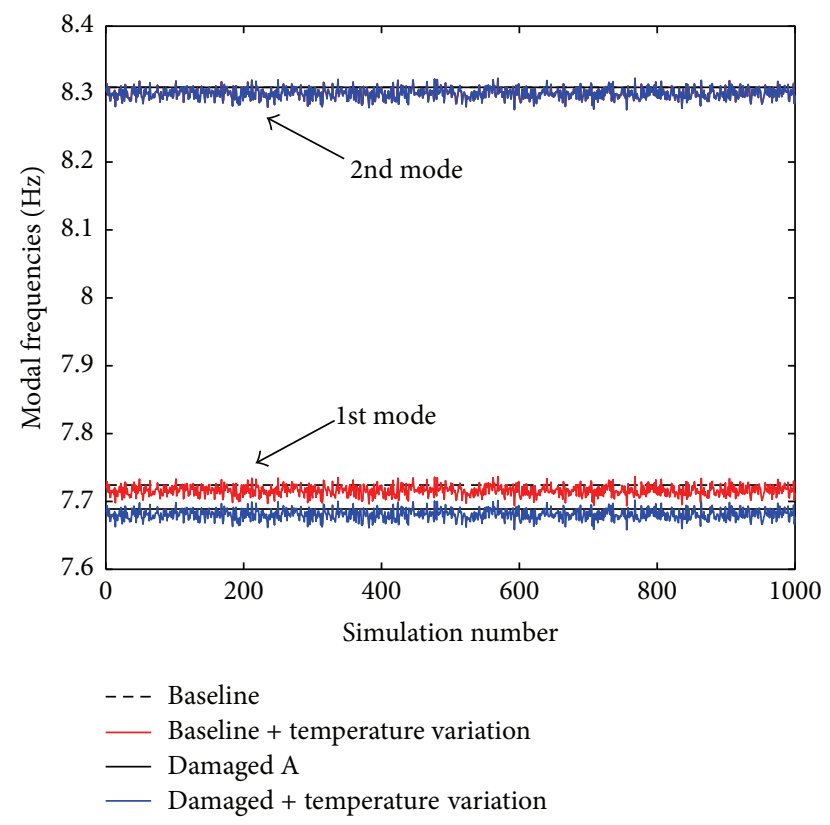

FIgURE 6: The first two modal frequencies for damage case A.

4.2. Damage Localization of the Structure under Different Temperature Variations. In this subsection, it is assumed that different parts of the platform structure are subject to different temperature variations. The structural members located above SWL (numbers 1-12) are subject to air temperature variation and structural members beneath SWL (numbers 13-36) are subject to water temperature variation. As described in Section 3.3, the air temperature is considered a Gaussian process with mean $10^{\circ} \mathrm{C}$ and standard deviation $8^{\circ} \mathrm{C}$, and the water temperature is considered a Gaussian process with mean $15^{\circ} \mathrm{C}$ and standard deviation $4^{\circ} \mathrm{C}$. In the following investigation, a 1000 Monte Carlo simulation is always conducted to investigate the effect of temperature variation on the accuracy of damage localization.

4.2.1. Damage Localization of Damage Case A. In damage case A, the damaged $y$-brace 12 , located above SWL, is a slanted diagonal structural element which is oriented in yoz plane. When the temperature variations are considered, the first two modal frequencies for damage case A under different conditions are illustrated in Figure 6. In Figure 6, the dotted and solid black lines are corresponding to the modal frequencies of the baseline and damaged structures, respectively, under constant temperature $\left(10^{\circ} \mathrm{C}\right)$, while the red and blue curves are associated with the baseline and damaged structures, respectively, after considering the temperature variations. From Figure 6 , one can see that a damaged $y$ brace would have negligible effect on the second mode shape that vibrates in the $x$ direction. Meanwhile, the temperature variation does have influence on the dynamic characteristics, as the modal frequencies of the structure after considering the temperature variation fluctuate around its counterpart. One can easily compute the percentage variation of the modal frequencies for the baseline structure after and before considering the temperature variations. The maximum percentage

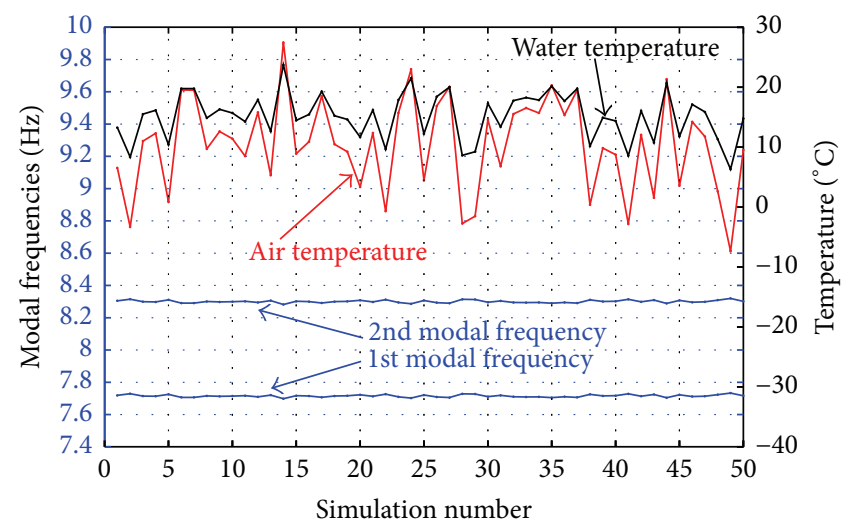

FIgURE 7: The first two modal frequencies in association with the water and air temperature for damage case A.

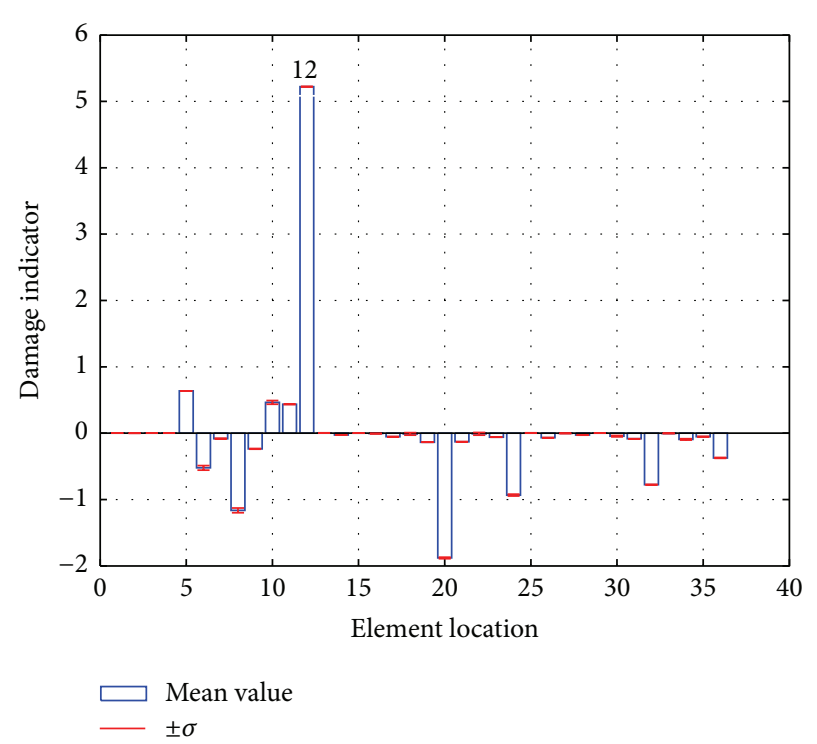

Figure 8: The damage indicator for damage case A when the temperature variation is considered.

change in the first modal frequency is about $-0.4 \%$, which is equivalent to that due to the inflicted damage in damage case A. At the same time, the first two modal frequencies in association with the water and air temperature of damage case A for the first 50 samples are illustrated in Figure 7. Generally, the modal frequencies will increase as the water and air temperature decreases.

When 1000 Monte Carlo simulation was used to investigate the effect of temperature variation on the accuracy of damage localization, the proposed damage indicator was calculated by using the proposed MSED method when the first two modes are utilized. Figure 8 shows the average and $\pm \sigma$ range ( $\sigma$ denotes the standard deviation) of the axial damage indicator $Z_{j}^{a}$ from the 1000 simulations, where each simulation is based on a temperature realization and the first two mode shapes are employed in the MSED method. From Figure 8 , it is obvious that the average damage indicator agrees perfectly well with their counterparts (results 


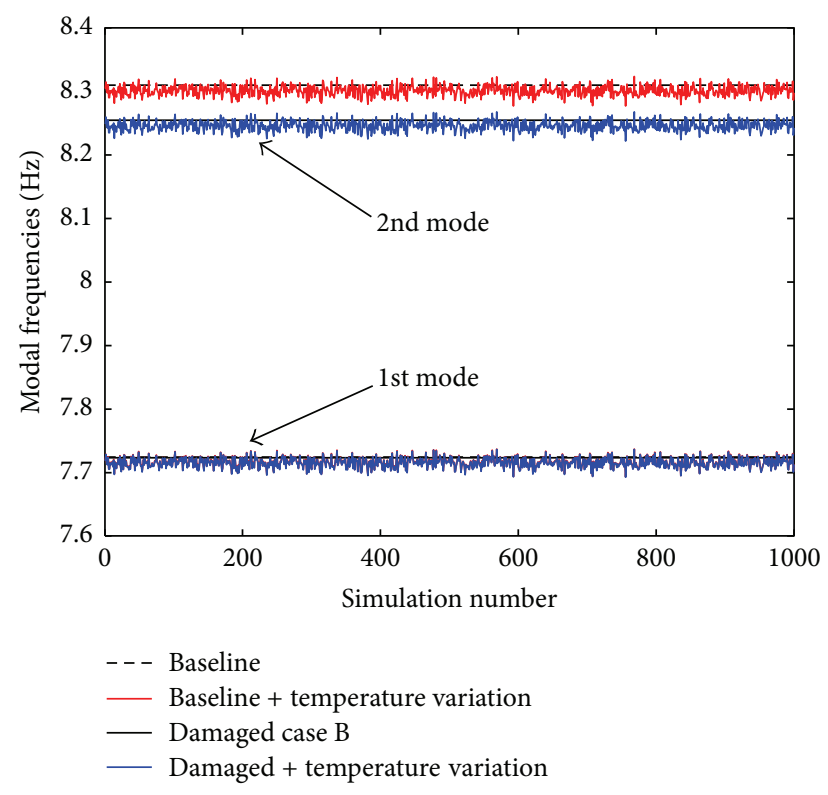

FIGURE 9: The first two modal frequencies for damage case B.

of Figure 4). At the same time, the standard deviation $\sigma$ corresponding to each structural element is negligible small, indicating that the MSED method is very robust to temperature variation.

4.2.2. Damage Localization of Damage Case B. In damage case $\mathrm{B}$, the damaged $x$-brace 18 , located beneath SWL, is a slanted diagonal structural element which is oriented in $x o z$ plane. The damage localization for damage case $B$ is to be investigated when the temperature variation is considered. The first two modal frequencies for damage case B under different conditions are illustrated in Figure 8. In Figure 8, the dotted and solid lines are corresponding to the modal frequencies of baseline and damaged structures under constant temperature $\left(10^{\circ} \mathrm{C}\right)$, respectively, while the red and blue curves are associated with the baseline and damaged structures, respectively, after considering the temperature variation. From Figure 9, one can see that a damaged $x$ brace would have negligible effect on the first mode shape that vibrates in the $y$ direction. Meanwhile, the temperature variation does have influence on the dynamic characteristics, as the modal frequencies of the structure after considering the temperature variation fluctuate around its counterpart.

Similar to damage case A, a 1000 Monte Carlo simulation was conducted to investigate the effect of temperature variation on the accuracy of damage localization. The temperature variation is identical to that used in damage case $A$ and the histogram of the simulated temperature is illustrated in Figure 3. After considering this temperature variation effect, the proposed damage indicator was calculated by using the MSED method when the first two modes are used. The average and $\pm \sigma$ range of the axial damage indicator $Z_{j}^{a}$ from the 1000 simulations are demonstrated in Figure 10, where each simulation is based on a temperature realization and

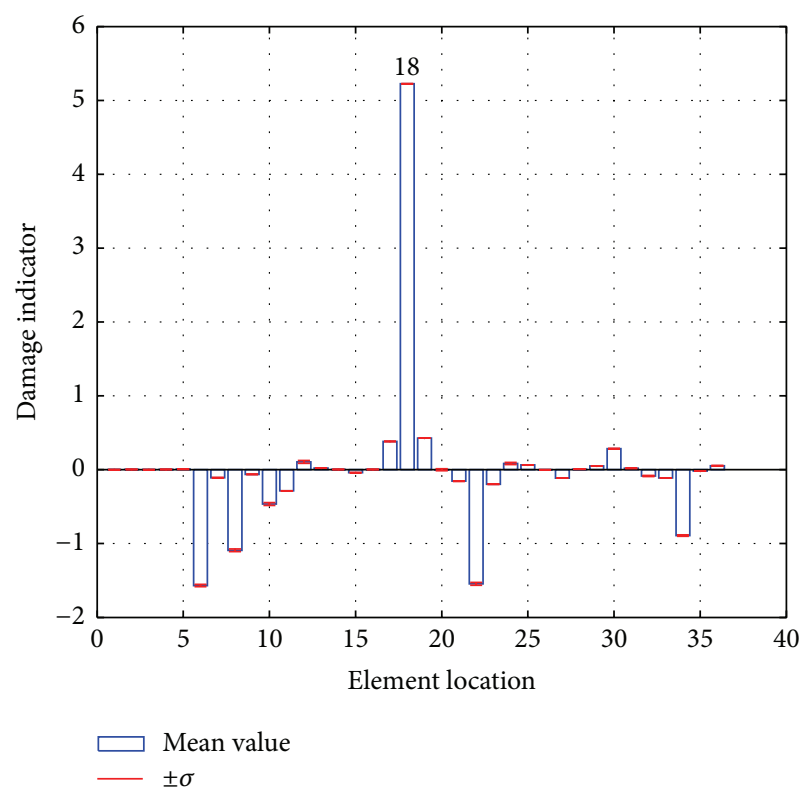

Figure 10: The damage indicator for damage case B when the temperature variation is considered.

the first two mode shapes are employed in the MSED method. From Figure 10, one can see that the average damage indicator associated with element 18 possesses the largest value, where the mean value is equal to 5.23 and $\sigma$ is 0.003 . At the same time, the average and standard deviation of the damage indicators corresponding to other structural members is negligibly small, meaning that the MSED method is able to locate the correct damage when the temperature variation is considered.

4.2.3. Damage Localization of Damage Case C. Damage case $\mathrm{C}$ is a double damage scenario, where a slanted $y$-brace 12 , located above SWL, and a slanted $x$-brace 18 , located beneath SWL, are assumed to be damaged. And the damage severities are $30 \%$ and $20 \%$, respectively. It is intended to investigate the effectiveness of the MSED method for locating multiple potential damages when the structure is subject to temperature variations. Figure 11 illustrates the first two modal frequencies for the double damage case $\mathrm{C}$ under different conditions. In Figure 11, the dotted and solid lines are corresponding to the modal frequencies of baseline and damaged structures under constant temperature $\left(10^{\circ} \mathrm{C}\right)$, respectively, while the red and blue curves are associated with the baseline and damaged structures, respectively, after considering the temperature variations. From Figure 10, it is obvious that the double damage has effects on both of the first two modal frequencies.

Similar to damage cases A and B, a 1000 Monte Carlo simulation was implemented to investigate the effect of temperature variations on the accuracy of damage localization. After considering the temperature variations, the proposed axial damage indicator was calculated by using the MSED method when the first two modes are used. The average and $\pm \sigma$ range of the damage indicator from the 1000 simulations 


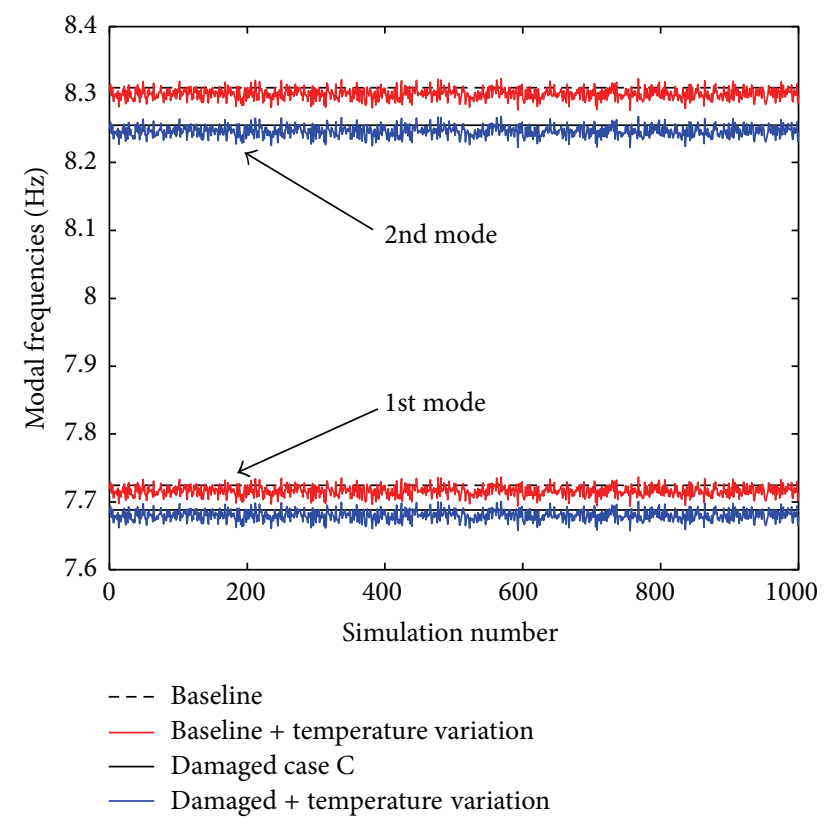

Figure 11: The first two modal frequencies for damage case C.

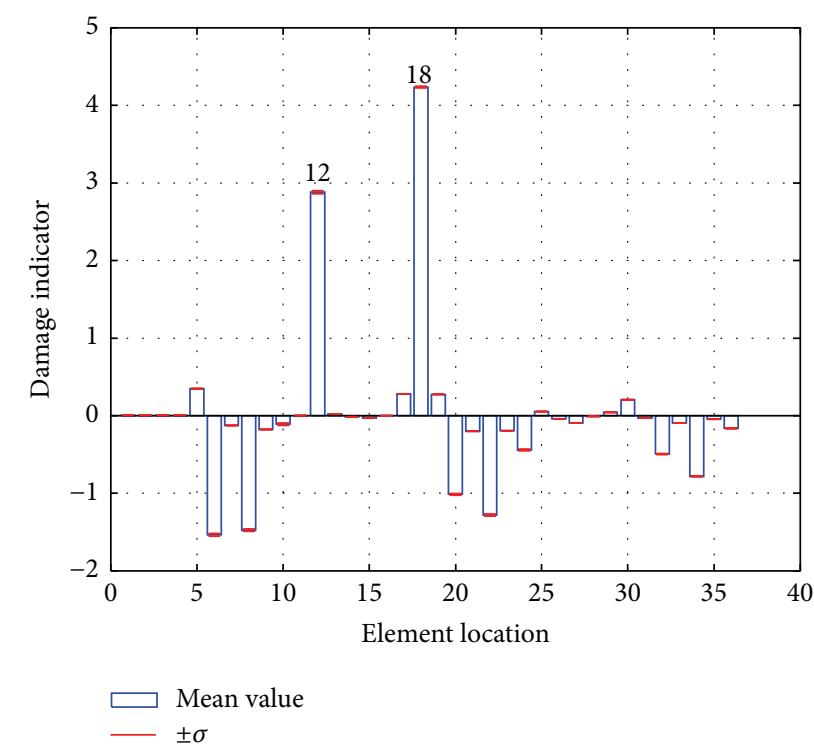

FIgUre 12: The damage indicator for damage case $\mathrm{C}$ when the temperature variation is considered.

are demonstrated in Figure 12, where each simulation is based on a temperature sample realization. From Figure 12, one can see that element 18 possesses the largest damage indicator, where the mean value is equal to 4.235 and $\sigma$ is 0.01 . And the second largest damage indicator corresponds to element 12 , with mean value 2.88 and $\sigma=0.02$, respectively. Average damage indicators corresponding to other structural members are negligibly small, meaning that the MSED method is able to locate the correct damage for double damage scenarios when different temperature variations are considered.

\section{Conclusions}

Vibration based damage detection is essentially important to ensure the safety of offshore structures. Offshore platforms are subject to environmental variations. Changes of modal parameters produced by environmental conditions can be equivalent or greater than the ones produced by damage. Therefore, development of vibration based damage detection methodology which is robust to environmental variation is essentially important for the structural safety. The present paper utilizes a recently developed modal strain energy decomposition (MSED) method to localize the damages of an offshore structure. The progress of the present paper takes the temperature variation into consideration and MonteCarlo simulation has been introduced to investigate the effect of temperature variation on the robustness of damage localization.

Numerical study is conducted on an offshore platform structure. Both single damage and double damage scenarios are considered. At the same time, different temperature variations associated with the structural members located in air and below water, respectively, are assumed. A Monte Carlo simulation is used to investigate the effect of temperature variation on the robustness of damage localization. The average and $\pm \sigma$ range ( $\sigma$ denotes the standard deviation) of the damage indicator from the 1000 simulations are statistically calculated for the three damage cases. Numerical simulation results show that the average damage indicator agrees perfectly well with their counterparts when the temperature variation is not considered, and the standard deviation $\sigma$ corresponding to each structural element is negligibly small, indicating that the MSED method is very robust to temperature variation. The proposed algorithm is capable of detecting the damage despite the temperature variations.

The present paper focuses on the direct effect of temperature variation on the material properties of the offshore structures and further clarifies its effect on damage localization. As change of modal parameters in an offshore structure would be a result of various factors, for example, changes in structural and water's physical properties, changes of annual still water level, and so forth, effect of the added mass variation (resulted from both temperature variation and still water level change) and the combined effects of added mass change and temperature induced structural material properties will be investigated in the near future.

\section{Conflict of Interests}

The authors declare that there is no conflict of interests regarding the publication of this paper.

\section{Acknowledgments}

The authors acknowledge the financial support by the National Basic Research Program of China (2011CB013704), National Natural Science Foundation of China (51379196), and the Major Program of the National Natural Science Foundation of China (51490675). 


\section{References}

[1] S. W. Doebling, C. R. Farrar, M. B. Prime, and D. W. Shevitz, "A summary review of damage identification methods that examine changes in dynamic properties," Shock and Vibration Digest, vol. 30, no. 2, pp. 91-105, 1998.

[2] A. Alvandi and C. Cremona, "Assessment of vibration-based damage identification techniques," Journal of Sound and Vibration, vol. 292, no. 1-2, pp. 179-202, 2006.

[3] P. Cawley and R. D. Adams, "The location of defects in structures from measurement of natural frequency," Journal of Strain Analysis, vol. 14, no. 2, pp. 49-57, 1979.

[4] O. S. Salawu, "Detection of structural damage through changes in frequency: a review," Engineering Structures, vol. 19, no. 9, pp. 718-723, 1997.

[5] S.-Q. Wang and H.-J. Li, "Assessment of structural damage using natural frequency changes," Acta Mechanica Sinica, vol. 28, no. 1, pp. 118-127, 2012.

[6] S. Wang, "Iterative modal strain energy method for damage severity estimation using frequency measurements," Structural Control and Health Monitoring, vol. 20, no. 2, pp. 110-120, 2013.

[7] G.-R. Gillich and Z.-I. Praisach, "Modal identification and damage detection in beam-like structures using the power spectrum and time-frequency analysis," Signal Processing, vol. 96, part A, pp. 29-44, 2014.

[8] W. M. West, "Illustration of the use of modal assurance criterion to detect structural changes in an orbiter test specimen," in Proceedings of the Air Force Conference on Aircraft Structural Integrity, Los Angeles, Calif, USA, February 1986.

[9] N. A. J. Lieven and D. J. Ewins, "Spatial correlation of mode shapes, the coordinate modal assurance criterion (COMAC)," in Proceedings of the 6th International Modal Analysis Conference, Kissimmee, Fla, USA, February 1988.

[10] J.-H. Kim, H.-S. Jeon, and C.-W. Lee, "Application of the modal assurance criteria for detecting and locating structural faults," in Proceedings of the 10th International Modal Analysis Conference, San Diego, Calif, USA, February 1992.

[11] O. S. Salawu and C. Williams, "Bridge assessment using forcedvibration testing," Journal of Structural Engineering, vol. 121, no. 2, pp. 161-173, 1995.

[12] N. Stubbs, J. T. Kim, and C. R. Farrar, "Field verification of a nondestructive damage localization and severity estimation algorithm," in Proceedings of the International Modal Analysis Conference. Society of Experimental Mechanics, Bethel, Conn, USA, February 1995.

[13] A. K. Pandey and M. Biswas, "Damage detection in structures using changes in flexibility," Journal of Sound and Vibration, vol. 169, no. 1, pp. 3-17, 1994.

[14] D. Bernal and B. Gunes, "Flexibility based approach for damage characterization: benchmark application," Journal of Engineering Mechanics, vol. 130, no. 1, pp. 61-70, 2004.

[15] B. Jaishi and W.-X. Ren, "Damage detection by finite element model updating using modal flexibility residual," Journal of Sound and Vibration, vol. 290, no. 1-2, pp. 369-387, 2006.

[16] J.-T. Kim and N. Stubbs, "Improved damage identification method based on modal information," Journal of Sound and Vibration, vol. 252, no. 2, pp. 223-238, 2002.

[17] H.-Z. Yang, H.-J. Li, and S.-Q. Wang, "Damage localization of offshore platforms under ambient excitation," China Ocean Engineering, vol. 17, no. 4, pp. 495-504, 2003.
[18] H. Li, H. Yang, and S.-L. J. Hu, "Modal strain energy decomposition method for damage localization in 3D frame structures," Journal of Engineering Mechanics, vol. 132, no. 9, pp. 941-951, 2006.

[19] S. Wang, F. Liu, and M. Zhang, "Modal strain energy based structural damage localization for offshore platform using simulated and measured data," Journal of Ocean University of China, vol. 13, no. 3, pp. 397-406, 2014.

[20] H. Sohn, K. Worden, and C. R. Farrar, "Statistical damage classification under changing environmental and operational conditions," Journal of Intelligent Material Systems and Structures, vol. 13, no. 9, pp. 561-574, 2002.

[21] Y. Xia, B. Chen, S. Weng, Y.-Q. Ni, and Y.-L. Xu, “Temperature effect on vibration properties of civil structures: a literature review and case studies," Journal of Civil Structural Health Monitoring, vol. 2, no. 1, pp. 29-46, 2012.

[22] G.-D. Zhou and T.-H. Yi, "A summary review of correlations between temperatures and vibration properties of long-span bridges," Mathematical Problems in Engineering, vol. 2014, Article ID 638209, 19 pages, 2014.

[23] H. Sohn, "Effects of environmental and operational variability on structural health monitoring," Philosophical Transactions of the Royal Society A, vol. 365, no. 1851, pp. 539-560, 2007.

[24] P. Moser and B. Moaveni, "Environmental effects on the identified natural frequencies of the Dowling Hall Footbridge," Mechanical Systems and Signal Processing, vol. 25, no. 7, pp. 2336-2357, 2011.

[25] A.-M. Yan, G. Kerschen, P. De Boe, and J.-C. Golinval, "Structural damage diagnosis under varying environmental conditions-part I: a linear analysis," Mechanical Systems and Signal Processing, vol. 19, no. 4, pp. 847-864, 2005.

[26] J. Kullaa, "Eliminating environmental or operational influences in structural health monitoring using the missing data analysis," Journal of Intelligent Material Systems and Structures, vol. 20, no. 11, pp. 1381-1390, 2009.

[27] H. J. Lim, M. K. Kim, H. Sohn, and C. Y. Park, "Impedance based damage detection under varying temperature and loading conditions," NDT and E International, vol. 44, no. 8, pp. $740-$ 750,2011

[28] E. J. Cross, K. Worden, and Q. Chen, "Cointegration: a novel approach for the removal of environmental trends in structural health monitoring data," Proceedings of the Royal Society A: Mathematical, Physical and Engineering Sciences, vol. 467, no. 2133, pp. 2712-2732, 2011.

[29] E. J. Cross, G. Manson, K. Worden, and S. G. Pierce, "Features for damage detection with insensitivity to environmental and operational variations," Proceedings of the Royal Society, vol. 468, no. 2148, pp. 4098-4122, 2012.

[30] B. Moaveni and I. Behmanesh, "Effects of changing ambient temperature on finite element model updating of the Dowling Hall Footbridge," Engineering Structures, vol. 43, pp. 58-68, 2012.

[31] D. M. Duggan, E. R. Wallace, and S. R. Caldwell, "Measured and predicted vibrational behavior of Gulf of Mexico platforms," in Proceedings of the 12th Annual Offshore Technology Conference, Houston, Tex, USA, May 1980.

[32] K. Fukunaga, Introduction to Statistical Pattern Recognition, Academic Press, New York, NY, USA, 1990. 
[33] S. Wang, "Damage detection in offshore platform structures from limited modal data," Applied Ocean Research, vol. 41, pp. 48-56, 2013.

[34] C. E. Woon and L. D. Mitchell, "Variations in structural dynamic characteristics caused by changes in ambient temperature: I. Experimental," in Proceedings of the 14th International Modal Analysis Conference, Dearborn, Mich, USA, February 1996. 


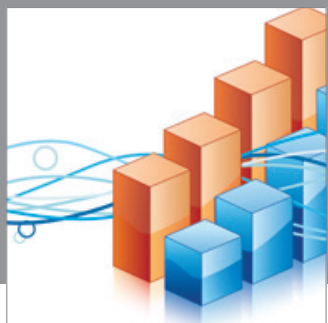

Advances in

Operations Research

mansans

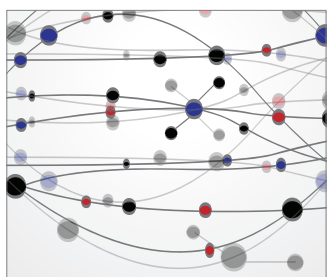

The Scientific World Journal
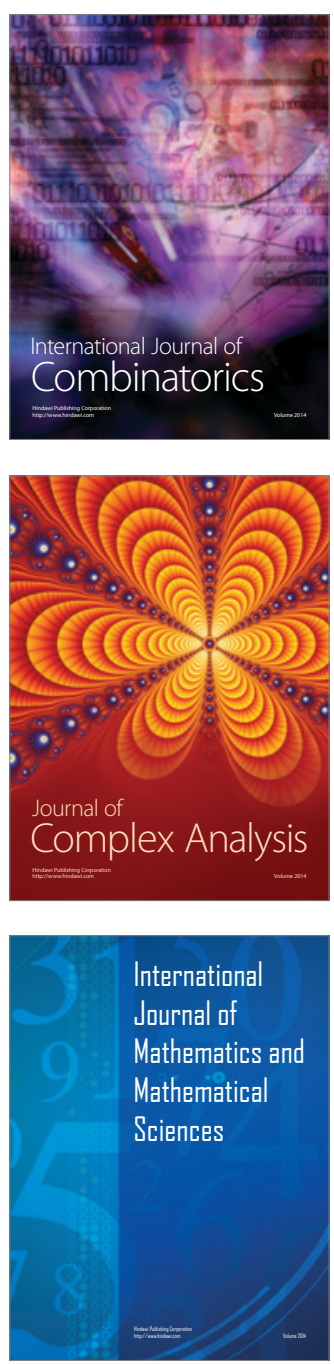
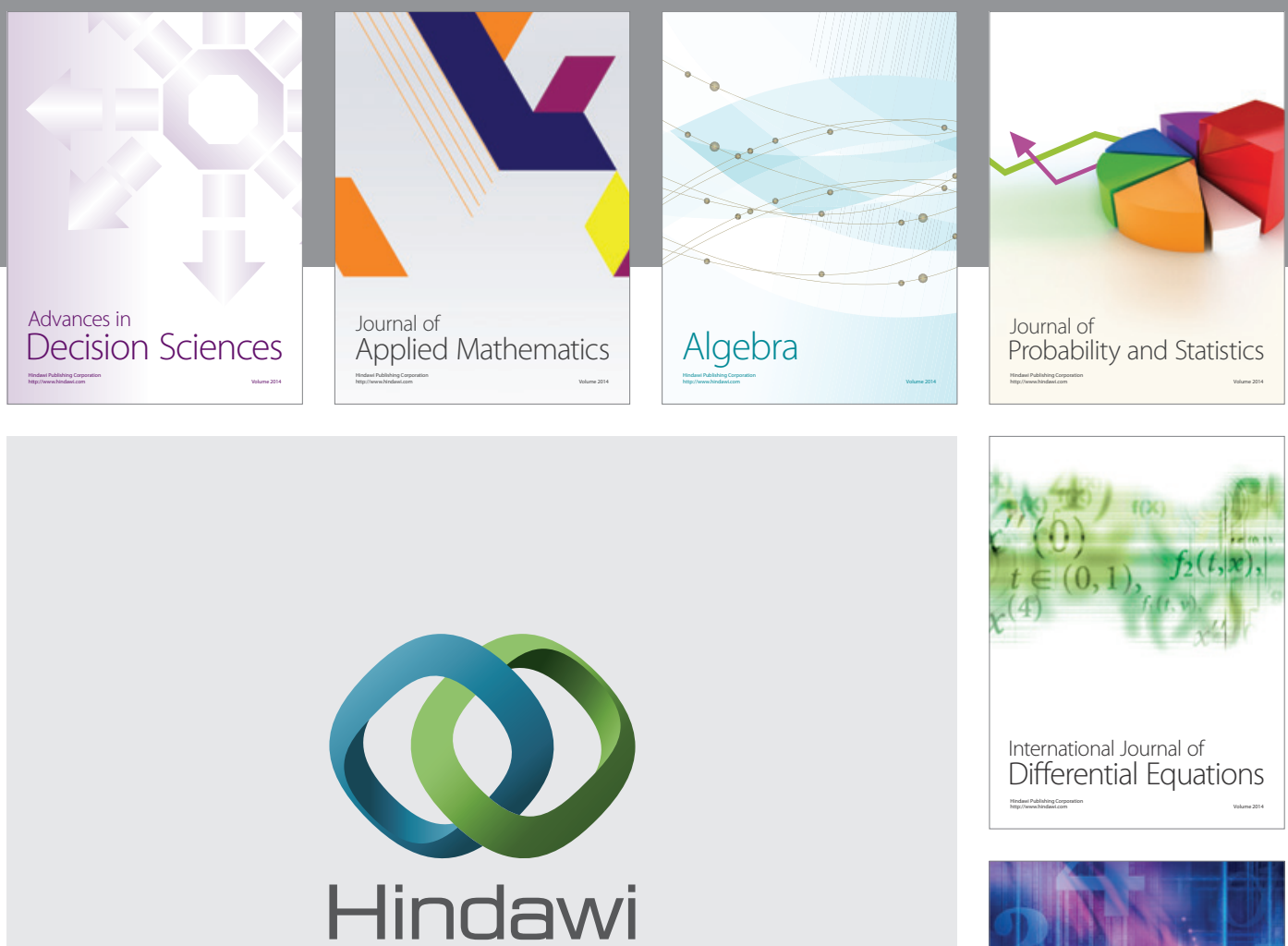

Submit your manuscripts at http://www.hindawi.com
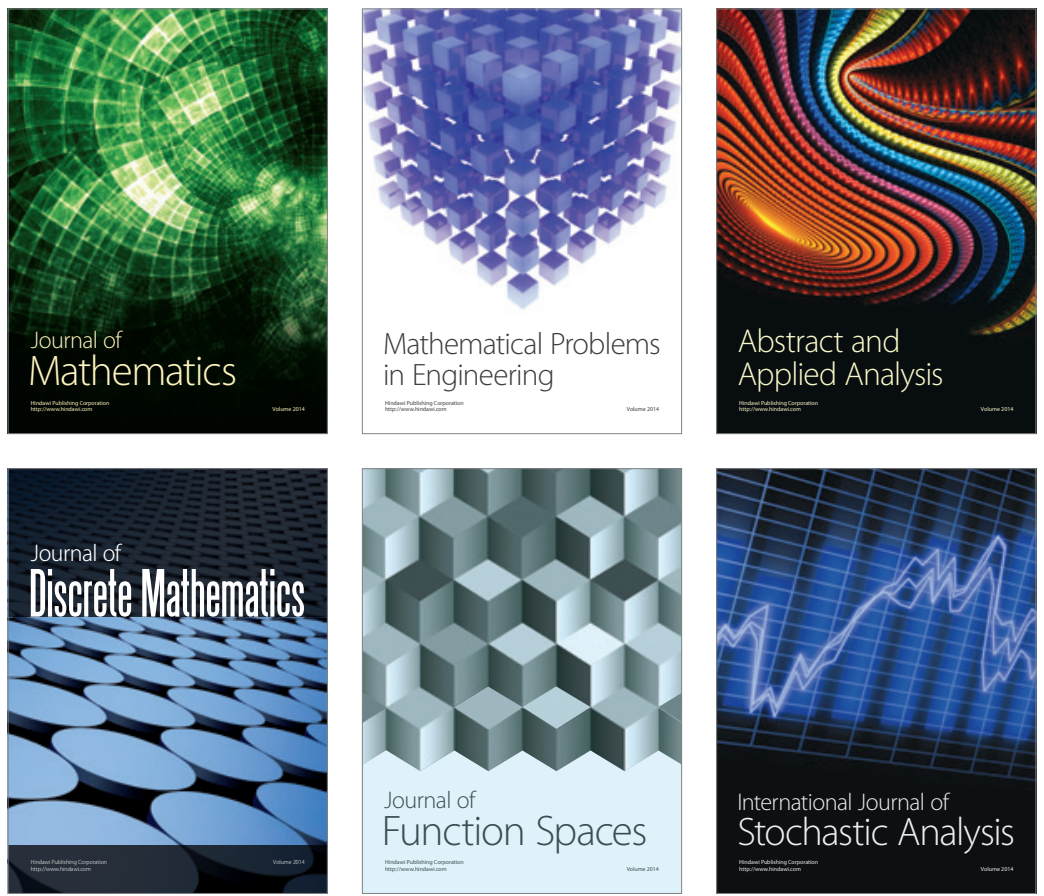

Journal of

Function Spaces

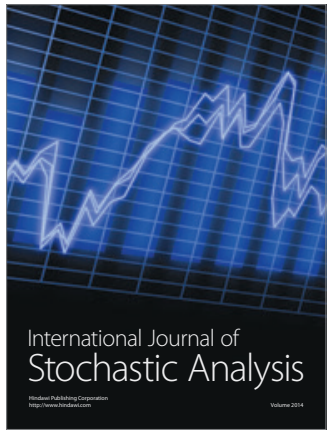

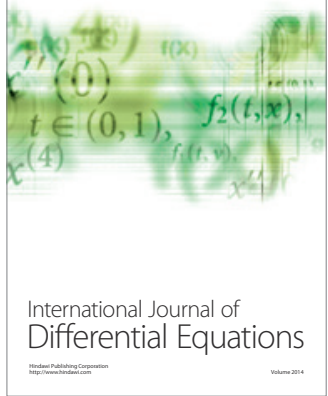
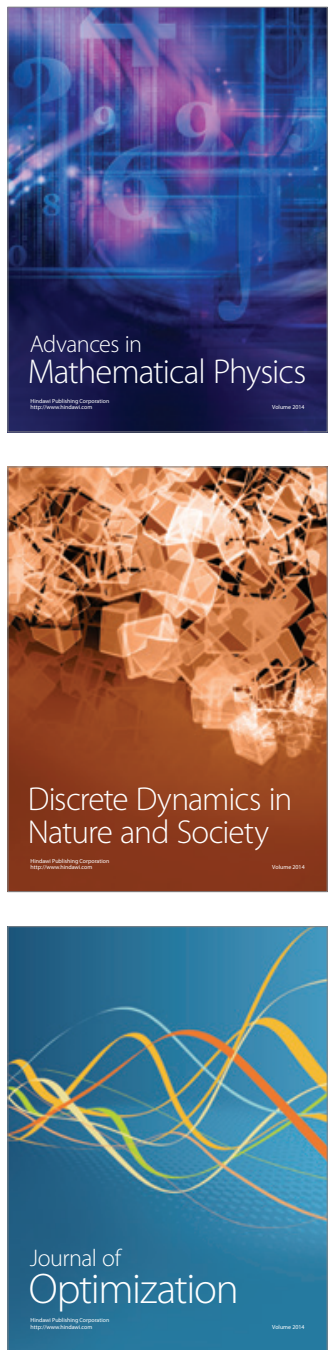